\title{
Optimizing Heterogeneous Cognitive Radio Networks by Rigorous Algorithms and Neural Networks
}

\author{
Mikio Hasegawa \\ Tokyo University of Science \\ 6-3-1 Niijyuku, Katsushika-ku \\ Tokyo 125-8585, Japan \\ $+81-3-5876-1717$ \\ hasegawa@ee.kagu.tus.ac.jp
}

\begin{abstract}
Cognitive radio technology improves efficiency of radio resource usage and quality of services in congested mobile networks, by reconfiguring the wireless connection settings according to the optimum decisions, which are made based on the collected context information. This paper focuses on the optimization algorithms for the decision-making to optimize radio resource usage in heterogeneous cognitive wireless networks. For the networks with centralized management, we can apply the algorithms to obtain rigorous solutions, because all of the network context information can be collected at the central manager. In order to avoid exponential increase of computational complexity in large-scale wireless networks, we model the target optimization problem as a minimum cost flow problem, and make it possible to solve the problem in polynomial time. In the networks with distributed management, the central network manager is not necessary, but the algorithms have to be run in a distributed manner. In order to optimize the defined objective function, we introduce the distributed energy minimization of the HopfieldTank neural network. We derive the decision-making rule for each terminal to optimize the entire network. We show validity of the proposed algorithms by numerical simulations.
\end{abstract}

\section{Keywords}

Cognitive radio, Optimization, Minimum cost flow problem, Radio resource management, Neural Networks

\section{INTRODUCTION}

Cognitive Radio Technology $[1,2]$ has been developed to improve radio resource usage of the wireless network environment. Recently, various wireless services have been widely deployed and the amount of the mobile traffic is continuously and rapidly increasing. To satisfy such a high demand to the mobile communications, the capacity of the mobile wireless networks has to be increased and some additional radio frequency bands are needed for them. However, the most of the frequency bands suitable for the mobile communication have already been assigned to the existing wireless services and the remaining bands are limited. Therefore, optimization of the radio resource usage of the Permission to make digital or hard copies of all or part of this work for personal or classroom use is granted without fee provided that copies are not made or distributed for profit or commercial advantage and that copies bear this notice and the full citation on the first page. To copy otherwise, to republish, to post on servers or to redistribute to lists, requires prior specific permission and/or a fee.

BIICT 2014, December 01-03, Boston, United States

Copyright @ 2015 ICST 978-1-63190-053-2

DOI 10.4108/icst.bict.2014.257894 wireless network is a very important issue in the current wireless networks.

The key idea of the cognitive radio is to efficiently utilize limited radio resources by dynamic spectrum access. In the conventional wireless systems, the radio access networks or the spectrum bands are statically assigned to the mobile systems. In such a case, some network or some frequency band may be highly congested, while some others have many available resources with small traffics. By dynamical use of the radio access networks or the spectrum bands, usage of limited radio resources can be optimized and the capacity and the quality of the wireless systems can be highly improved.

In the original definition of the cognitive radio [1,2], it has been defined as a kind of cognitive dynamic systems [3]. The cognitive radio systems observe and recognize radio network environment, make reconfiguration decisions, and apply the corresponding action to reconfigure the network. By this approach, various kinds of radio parameters in the wireless communication systems can be optimized by appropriate actions. By learning relation between the actions and the improvement of the performance, its recognition part will be improved with increase of the samples. For some of the performance factors, the relation between the actions and the performance improvement can be approximately predefined. The best decision can be selected by solving an optimization problem, which can be formulated based on the relation between the actions and the performance improvement. In this paper, we focus on the optimization algorithm for the best decision. We deal with the latter case of the cognitive radio system and discuss optimization algorithms.

There are various optimization problems, which can be defined to improve the radio resource usages [4]-[8]. It depends on the observable factors and tunable parameters of the system. In this paper, we deal with the problem in heterogeneous wireless networks, in which various different types of wireless services coexist. As one of the standards to realize such a cognitive radio system, IEEE Std. 1900.4 [9] includes the distributed radio resource usage optimization (DRRUO) as a use case of its defined cognitive radio system. The terminals select the best radio resource from various types to optimize the efficiency of the radio resource usage. In order to make the optimal decision, necessary information can be collected using the architecture and protocol defined in IEEE1900.4.

In this paper, we focus on optimization techniques to maximize the efficiency of the radio resource usage in heterogeneous wireless networks. First, we define an optimization problem of load balancing, which improves service quality of the 
IP-based heterogeneous wireless networks. Second, under the assumption that the entire network can be managed at a centralized server based on IEEE1900.4, we develop an optimization algorithm to obtain rigorous solution in short time. Third, since such a centralized management becomes difficult for huge-scale networks, we develop a distributed optimization algorithm based on the theory of Hopfield-Tank neural networks [10].

\section{HETEROGENEOUS COGNITIVE RADIO NETWORKS AND ITS OPTIMIZATION PROBLEM}

Recently, several wireless communication standards based on the idea of the cognitive radio have been developed. In IEEE802, wireless LANs, 802.11af [11], wireless broadband systems, 802.22 [12] and IEEE802.16h[13], have been developed as the real services using the cognitive radio technology, which utilizes white spaces of the TV spectrum bands. Such cognitive radio systems obtain available spectrum resource information and select the best one from them with avoiding interferences to the primary wireless systems, TV broadcasting.

On the other hand, optimal selection of the best wireless service also improves quality of wireless services by efficient radio resource usage defined as the DRRUO in IEEE1900.4 [9]. In such wireless networks, when the improvement of the quality can be estimated by the collected information, the selection of the most appropriate action can be defined as an optimization problem.

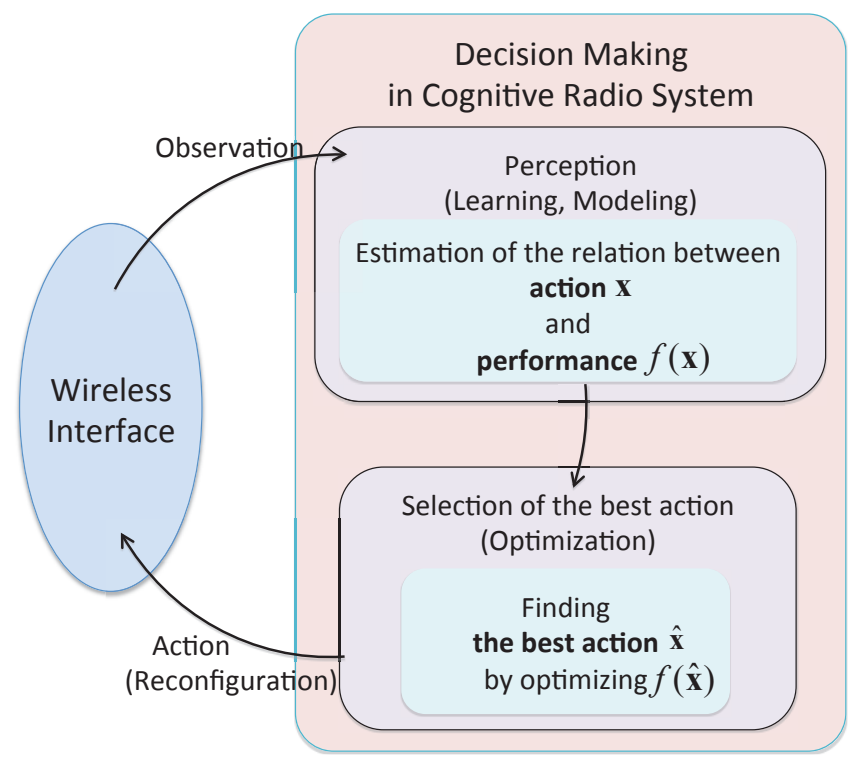

Figure 1. A cognitive radio system.

Figure 1 shows a general cognitive radio system, which can be applied to the systems described above. It observes the state of the cognitive radio system, estimates the relation between the action and performance improvement, and finds the best action based on the estimated relation. Generally, the relation between the action and the corresponding performance, $f(\mathbf{x})$, is unknown, and it has to be estimated by some learning algorithm. Selection of the best action can be achieved by searching the optimal state of $f(\mathbf{x})$, that corresponds to solving the optimization problem. In this paper, we focus on this part, selection of the best action, by optimization algorithms.

There are two approaches to optimize the network. One is to calculate the optimal state at a centralized server, which manages all of the wireless connections. For such case it is possible to rigorously optimize the entire network. The drawback of such centralized schemes is that it is necessary to collect all of the information at the center, which may generate the overheads for exchanging the context information and the control information of entire networks. Therefore, as the second, we consider to apply distributed optimization algorithms, which run in parallel, for the large-scale network optimization.

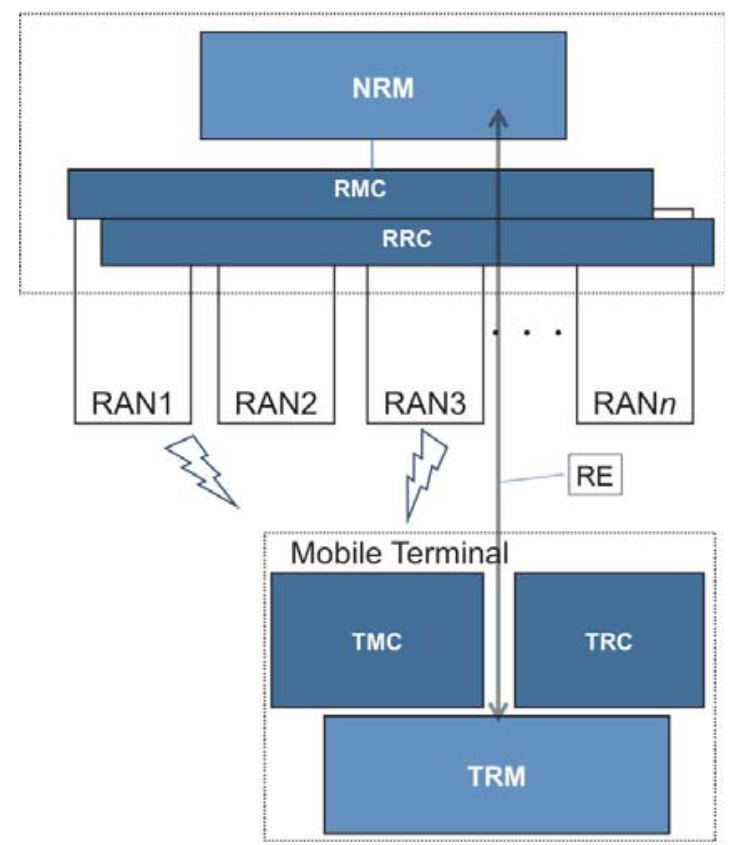

Figure 2. Management archtecture of IEEE1900.4 to optimize radio resource usage in heterogeneous wireless networks [9].

As a cognitive radio system, which can be optimized by those optimization algorithms, IEEE1900.4 defines the architecture to exchange the context information and the spectrum selection policy between the terminal side and the network side [9], whose management architecture is shown in Fig. 2. The network reconfiguration manager (NRM) collects context information of the radio access networks (RAN) via the RAN measurement collector (RMC). The terminal reconfiguration manager (TRM) collects the terminal context information by the terminal measurement collector (TMC). The collected information at the NRM and the TRM are exchanged between the terminal side and the network side, via the radio enabler (RE). After the information collection, the NRM and the TRM can make decision for reconfiguration. The action for the selected reconfiguration will be taken using the RAN reconfiguration controller (RRC) for the network side and the terminal reconfiguration controller (TRC) at the terminal side. Using this architecture, an optimization algorithm for the best decision making can be calculated at either at the NRM or at the TRM. For the centralized optimization, the NRM will collect all of the information, make 
decision, and notify the best action for all of the TRMs on the terminals. For the distributed optimization, the TRMs on the terminals will collect necessary context information via the NRM, and make the best decision at themselves.

In this paper, we focus on the optimization algorithms for the best action selection in heterogeneous cognitive wireless networks. As a typical example of optimization problems for the cognitive radio networks, we deal with the avoidance of the traffic congestions by the load balancing. In the IEEE1900.4 standard document [9], the load balancing is defined as a typical example use case to improve the radio resource usage.

There may be several ways to formulate the optimization problem of load balancing. In this paper, we formulate the objective function, based on several assumptions that should be introduced for optimization of the heterogeneous wireless networks.

The first assumption is that the cognitive wireless network is heterogeneous. Recently, various kinds of wireless networks are available, such as the cellular phone system, wireless LAN, wireless broadband systems and so on, to connect to the Internet. Recent mobile terminals are equipped with several wireless modules to connect several of those heterogeneous wireless networks. The capacities of the networks are different among those systems. In such heterogeneous wireless networks, a high capacity network can accept more terminals for the same congestion level. Therefore, the optimum state of the traffic load balancing should be defined so that all of the communicating terminals will have the same throughput to transmit packets. Since the communicating terminals will utilize all of the network resources, whose entire capacity is constant, $\sum_{j=1}^{n} c_{j}=\sum_{i=1}^{m} T_{i}=$ const. will be satisfied, where $c_{j}$ is the capacity of the $j$ th base station, $T_{i}$ is the available throughput for the $i$ th terminal, $n$ is the number of the base stations, and $m$ is the number of the communicating terminals, respectively. Therefore, the optimum state of the load balancing in the heterogeneous network can be obtained by minimizing

$F_{\text {OBJ }}(\mathbf{T})=\sum_{i=1}^{m} \frac{1}{T_{i}}$,

with satisfying $\sum_{i \in \mathbf{S}_{j}} T_{i}<c_{j}$ for all $j$, where $S_{j}$ is a set of the terminals connecting to the $j$ th base station.

The second assumption is that those wireless networks are IP-based. Recent wireless services, such as the wireless LAN, LTE and so on, are packet-based networks, which share limited radio resources with many terminals. For example, in the wireless LAN systems, the transmission opportunities for the mobile terminals are almost equal by highly fair MAC protocol. Therefore, the throughput of each terminal can be assumed as $T_{i}=\frac{c_{L(i)}}{\left|\mathbf{S}_{L(i)}\right|}$ for such networks, where $\left|\mathbf{S}_{j}\right|$ is the number of mobile terminals connecting to the base station $j$, and $L(i)$ is the base station to which the terminal $i$ is connecting, respectively.

Based on the above assumptions, the traffic load-balancing problem in heterogeneous IP-based wireless networks can be formulated as minimization of
$F_{\mathrm{OBJ}}(\mathbf{L})=\sum_{i=1}^{m} \frac{\left|\mathbf{S}_{L(i)}\right|}{c_{L(i)}}$,

under the condition that all the communicating terminal $i$ are located in the service area of its selected base station $L(i)$, $L(i) \in \mathbf{A}_{i}$, where $\mathbf{A}_{i}$ is a set of available base stations for the terminal $i$.

This objective function is of the vector $\mathbf{L}$, which is the list of the connecting base station of each communicating mobile terminal. In this combinatorial optimization problem, the number of combinations becomes $\mathrm{m}^{n}$, and it becomes very hard to solve when the number of base stations increases. For such a problem having combinatorial explosion, it is difficult to find the optimum state. Therefore, we usually give up to find the optimum solution and try to find good approximate solution. In the next section, we show that this problem can be rigorously solved with small computational amount.

\section{OPTIMIZING COGNITIVE RADIO NETWORKS BY A CENTRALIZED ALGORITHM}

In the IEEE 1900.4 architecture [9], it is possible to optimize the heterogeneous wireless network usage by computation at the centralized manager, NRM. Various kinds of the network context information can be collected to the NRM, which can solve the optimal action for the entire wireless network by optimization algorithms. The selected optimal action will be notified from the NRM to the TRMs of each terminal device, which will switch the selection of the network to the appropriate one.

In the combinatorial optimization problem in Eq. (2) to find the optimum base station selections $\mathbf{L}$, the number of combinations increases exponentially with the increase of the number of base stations. In this section, we show that the problem can be solved rigorously by the following algorithm even for large-scale heterogeneous wireless networks.

\subsection{A Rigorous Algorithm to Solve Exactly Optimum State of Cognitive Radio Networks}

Our proposed approach is to formulate the combinatorial optimization problem as a network flow problem with modeling a graph. We transform the heterogeneous base station selection problem in Eq. (2) to a minimum cost flow problem, which can be rigorously solved by small computational complexity.

The minimum cost flow problem is to find the optimal flow $z_{e}$ for each edge $e$ in the graph $G, e \in E(G)$, with minimizing the entire cost in a directional graph. It is minimization of

$$
F_{\mathrm{MCF}}(\mathbf{z})=\sum_{e \in E(G)} u_{e} z_{e}
$$

with satisfying $z_{e}<p_{e}$, where $u_{e}$ is the cost, and $p_{e}$ is the capacity of the edge $e$. Some of the vertices in the graph $G$, have the supply or the demand of some amount of the flow, which is defined as $b_{v}$. When $b_{v}>0$, the vertex v supplies the output flow whose amount is $\left|b_{v}\right|$, while when $b_{v}<0$, the vertex $v$ demands the input flow amount $\left|b_{v}\right|$. All of the flow $z_{e}$ should be integer numbers in this problem. There are several optimization algorithms to obtain the exact minimum value of Eq. (3) by small computation complexity 
[14] - [17]. In this paper, we formulate the base station selection problem defined in the previous section as the minimum cost flow problem to obtain the exactly optimum solution even for the largescale networks.

Based on the assumption that the base station of the IP-based wireless network gives fair transmission timing to each connecting terminals, the throughput for the terminals connecting to the same base station becomes equal. Therefore, the objective function in Eq. (2) can be transformed to the following form using the set of the connecting terminal to the $j$ th base station, $\mathbf{S}_{j}$ :

$$
F_{\mathrm{OBJ}}=\sum_{i=1}^{m} \frac{1}{T_{i}}=\sum_{j}^{n} \frac{1}{T_{\mathbf{S}_{j}}} \cdot\left|\mathbf{S}_{j}\right|=\sum_{j}^{n} \frac{1}{c_{j}}\left|\mathbf{S}_{j}\right|^{2}
$$

This objective function is also optimized by the selection of the links between the terminals and the base stations. Since the $\left|\mathbf{S}_{j}\right|$ can be regarded as the flow between the base station $j$ and the global network, we formulate the minimization problem in Eq. (4) as a minimum cost flow problem, which can be rigorously solved without exponential increase of the computation amount.

In order to formulate this problem as the minimum cost flow problem shown in Eq. (3), we need to remove the square term, $\left|\mathbf{S}_{j}\right|^{2}$ of Eq. (4). Using $\left|\mathbf{S}_{j}\right|^{2}=\sum_{l=1}^{\left|\mathbf{S}_{j}\right|} 2 l-1$, we modify Eq. (4) to the following form:

$$
F_{\text {OBJ }}=\sum_{j}^{n} \frac{1}{c_{j}} \sum_{l=1}^{\left|\mathbf{S}_{j}\right|} 2 l-1=\sum_{j}^{n} \sum_{l=1}^{\left|\mathbf{S}_{j}\right|} \frac{2 l-1}{c_{j}} .
$$

To minimize this equation, we design a graph shown in Fig. 3 . The cost and the capacity are expressed as $\left(w_{i j}, p_{i j}\right)$ for each edge. The vertices $v_{i}^{M}$ correspond to the $i$ th mobile terminals, and the vertices $v_{j}^{B}$ to the $j$ th base stations, respectively.

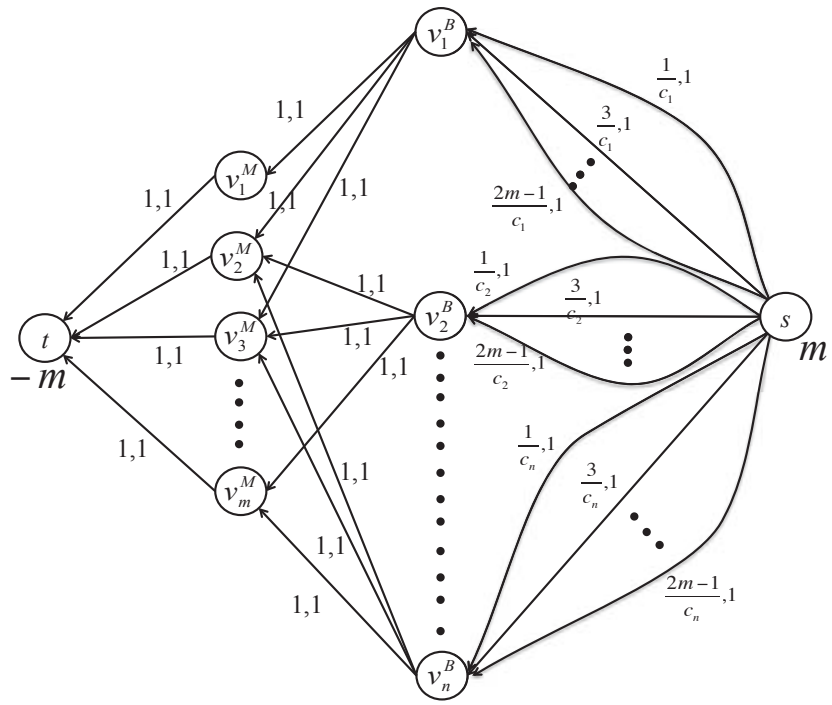

Figure 3. Load-balancing problem formulated as a minimum cost flow problem.

The supplied flow from the source $\mathrm{s}$ and the demanded flow by the sink $t$ are set at the number of mobile terminals $m$. The edges between the sink $t$ and all of the $v_{i}^{M}$ are set at $(1,1)$, since it is assumed that each terminal establishes a wireless link with one base station for each. This means that the flows $z_{v_{i}^{M}, t}$ on all edges between the sink $t$ and all of the $v_{i}^{M}$ becomes 1 . Also for the edges between the vertices $v_{i}^{M}$ and $v_{j}^{B}$, we set $(1,1)$, for the pairs that the base station $j$ is available for the communicating terminal $i, j \in \mathbf{A}_{i}$. Since each $v_{i}^{M}$ has one exit, which goes to the sink $t$, exactly one edge from vertices $v_{j}^{B}$ to $v_{i}^{M}$ will have flow 1 , and the selected edge having this flow will be the connection between the base station and the communicating terminals, which should be established in the optimal state. The cost calculated in Eq. (5) is added in the edges between the source vertex $s$ and the vertices $v_{j}^{B}$, which have cost $\frac{2 l-1}{c_{j}}$. When $\left|\mathbf{S}_{j}\right|$ terminals selected the base station $j,\left|\mathbf{S}_{j}\right|$ edges having smaller costs, $\frac{1}{c_{j}}, \frac{3}{c_{j}}, \ldots, \frac{2\left|\mathbf{S}_{j}\right|-1}{c_{j}}$, will be selected by minimization of the total cost. Therefore, the transformed objective function, $\sum_{j}^{n} \frac{1}{c_{j}}\left|\mathbf{S}_{j}\right|^{2}$, can be minimized by the selection of the optimal wireless links between the terminals and base stations.

\subsection{Computational Complexity}

In order to obtain the solution of this minimum cost flow problem for RAN selection, we use the algorithm in Ref. [17], which solves the exact optimum solution with small computational complexity whose order is $O\left(N_{v} N_{e} \log \left(N_{v} C\right)\right)$, where $N_{v}$ and $N_{e}$ are the numbers of the vertices and the edges, and $C$ is the maximum cost on the edges. In the minimum cost flow problem for the base station selection designed in Fig. 3, it becomes $O(m(m+n+2)(m+n+1) \log (C(m+n+2)))$. In the originally defined combinatorial optimization problem of load balancing for the packet based heterogeneous wireless networks in Eq. (2), the number of combinations was $m^{n}$, which increases exponentially. It is difficult to obtain the exact solution of such combinatorial optimization problems whose size is large-scale. Our proposed algorithm clarifies that such a problem with combinatorial explosion is not always NP-hard and we can obtain the exact solution by small computational amount. The proposed scheme does not require large computational load even for very large problems.

We evaluate the increase of the computational time of the proposed scheme by computer simulations. Figure 4 shows the CPU time required for obtaining the rigorous solutions by our proposed scheme with changing the number of base stations and terminals. We run the scheme on Solaris 10 installed in a server computer with floating point operating at $2926 \mathrm{MHz}$.

The results in Fig. 4 clarify that the proposed scheme can obtain rigorous solutions in short time, even for large-scale networks. In the largest case, 500 base stations and 900 mobile terminals, only 0.036 seconds was required to obtain the rigorous solution. Increase of the computational time is not exponential, but almost proportional to the number of the mobile terminals or the number of base stations. In the maximum case with 900 terminals, the required time was about 0.008 seconds for the 100 base stations, while it was about 0.036 seconds for the 500 base 
stations case. This means that we do not have to use heuristic algorithms, which can only obtain approximate solutions, for network load balancing, when the centralized server can manage all of heterogeneous wireless connections of the terminals.

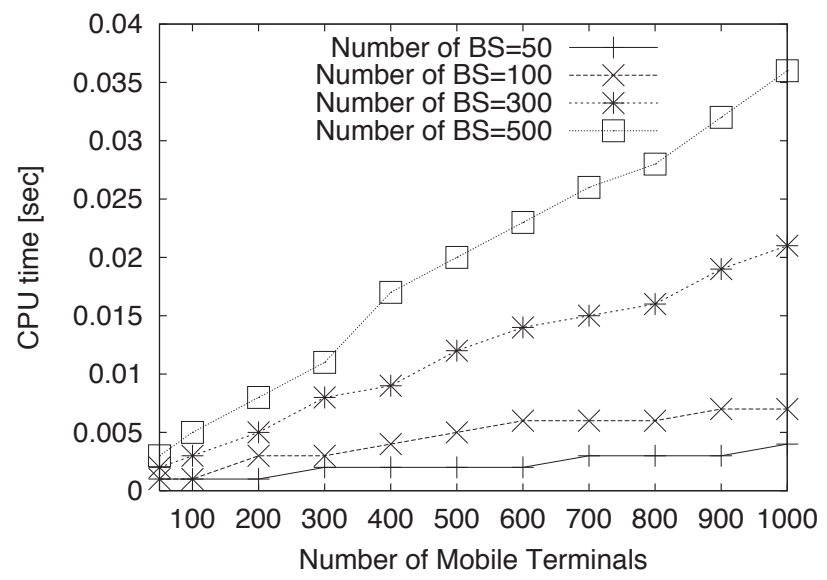

Figure 4. CPU time to obtain rigorous solutions of BS selection problem for large scale wireless networks.

\section{OPTIMIZING COGNITIVE RADIO NETWORKS BY A NEURAL NETWORK BASED DECENTRALIZED ALGORITHM}

In the previous section, we showed that it is possible to solve the exactly optimum state of the heterogeneous wireless network, by modeling the problem as a minimum cost flow problem. However, it is not easy to develop a centralized management network for the large-scale wireless systems, in which a centralized server manages a huge number of wireless links with notifying the optimum selection to all of the communicating terminals. Therefore, in this section, we consider the case that there is no centralized server to compute the optimum state and the computation have to be done in a distributed method.

As a distributed optimization algorithm to minimize the objective function, we introduce the Hopfield-Tank neural network [10]. It is a mutually connected neural network, in which each neuron updates their state by the following equations:

$x_{i j}(t+1)=\left\{\begin{array}{cc}1 & \text { when } \sum_{k=1}^{m} \sum_{l=1}^{n} w_{i j k l} x_{k l}(t)>\theta_{i j}, \\ 0 & \text { otherwise }\end{array}\right.$

where $x_{i j}(t)$ is the state of the $(i, j)$ th neuron at time $t, w_{i j k l}$ is the connection weight between the $(i, j)$ th and $(k, l)$ th neurons, and $\theta_{i j}$ is the threshold of the $(i, j)$ th neuron.

In this neural network, by setting the same weights for the both directions of each connection, $w_{i j k l}=w_{k l i j}$, zero weights for the self-connections, $\quad w_{i j i j}=0$, and updating each neuron asynchronously, the update of the neural network will converge to some state within some iterations. At each update of this neural network, the energy function

$$
E(\mathbf{x})=-\frac{1}{2} \sum_{i=1}^{m} \sum_{j=1}^{n} \sum_{k=1}^{m} \sum_{l=1}^{n} w_{i j k l} x_{i j} x_{k l}+\sum_{i=1}^{m} \sum_{j=1}^{n} \theta_{i j} x_{i j}
$$

always decreases. The state the neural network converges to a state corresponds to a minimum of the energy function $E(t)$ by updating by Eq. (6). It means the simple neuronal update can make a distribute search of a state $\mathbf{x}$ corresponding to the minimum of the energy function $E(\mathbf{x})$.

In previous researches, this minimization property has been applied to combinatorial optimization problems [10]. First, the state of the optimization problem should be defined by the state of neurons $x_{i j}$ and the objective function has to be expressed as a function of the neuronal states. By comparing the function with the energy function of the neuronal state in Eq. (7), the connection weight $w_{i j k l}$ and the threshold $\theta_{i j}$ can be calculated. By updating neurons using Eq. (6) with those $w_{i j k l}$ and $\theta_{i j}$, the state of the neural network corresponding to the minimum value of the energy function can be autonomously obtained by distributed neuronal updates. This minimum corresponds to the minimum of the objective function of the original problem and the optimum state will be obtained by checking the state of the neurons.

In this paper, we show a scheme to optimize the base station selection problem by this distributed optimization algorithm. We defined the state matrix $\mathbf{x}$ of the neural network for the connection matrix between the terminals and base stations. $\mathbf{x}$ is defined only for the available connections, $x_{i j} \in \mathbf{A}_{i}$. When the terminal $\mathrm{i}$ is connecting to the base station $j$ at time $t, x_{i j}(t)=1$. Otherwise, $x_{i j}(t)=0$. By using $x_{i j}$, the number of terminals connecting to the base station $j$, which is $\left|\mathbf{B}_{j}\right|$, can be counted by

$\left|\mathbf{B}_{j}\right|=\sum_{k=1}^{m} x_{k j}$.

Since each communicating terminal selects only one base station for each, only one $x_{i j}$ among $x_{i 1}, \ldots, x_{i n}$ becomes 1 , corresponding to the selected base station $j$ by the terminal $i$. Therefore, $\frac{\left|\mathbf{B}_{L(i)}\right|}{c_{L(i)}}$ for the terminal i can be determined using $x_{i j}$ as

$\frac{\left|\mathbf{B}_{L(i)}\right|}{c_{L(i)}}=\sum_{j=1}^{n} \frac{\left|\mathbf{B}_{j}\right|}{c_{j}} x_{i j}$.

Using Eqs. (8) and (9), the problem defined in Eq. (2) can be transformed to minimization of

$$
\begin{aligned}
F_{\text {OВJ }}(\mathbf{x}) & =\sum_{i=1}^{m} \sum_{j=1}^{n} \frac{1}{c_{j}} x_{i j} \sum_{k=1}^{m} x_{k j} \\
& =\sum_{i=1}^{m} \sum_{j=1}^{n} \sum_{k=1}^{m} \frac{1}{c_{j}} x_{i j} x_{k j},
\end{aligned}
$$

under the constrain that $x_{i j}=0$ for $j \notin \mathbf{A}_{i}$.

In order to minimize this objective function by the neuronal updates, the objective function defined in Eq. (10) is transformed to the following form of the energy function: 
$F_{\mathrm{OBJ}}(\mathbf{x})=\sum_{i=1}^{m} \sum_{j=1}^{n} \sum_{k=1}^{m} \sum_{l=1}^{n} \frac{1}{c_{j}} \delta_{j l} x_{i j} x_{k l}$

where $\delta_{i j}$ is the Kronecker delta, $\delta_{i j}=1$ when $i=j, \delta_{i j}=0$ otherwise. Here, we have to care that the self-connection weight should be 0, but there are coefficient on $x_{i j} x_{k l}$ in this form of Eq.

(11). In order to remove the self-connections, we transform this equation to the following form, by using $x_{i j} x_{i j}=x_{i j}$ because $x_{i j}$ takes the values 0 or 1 only:

$$
\begin{aligned}
F_{\mathrm{OBJ}}(\mathbf{x})=\sum_{i=1}^{m} \sum_{j=1}^{n} \sum_{k=1}^{m} \sum_{l=1}^{n} \frac{1}{c_{j}}\left(1-\delta_{i k}\right) \delta_{j l} x_{i j} x_{k l} \\
+\sum_{i=1}^{m} \sum_{j=1}^{n} \frac{1}{c_{j}} x_{i j} .
\end{aligned}
$$

Comparing the coefficients of the neuron states $x_{i j}(t)$ in this form of the objective function with the energy function of the neural network in Eq. (7), the connection weight $w_{i j k l}$ and the threshold $\theta_{i j}$ to minimize the objective function can be obtained as follows:

$w_{i j k l}=-2 \frac{1}{c_{j}}\left(1-\delta_{i k}\right) \delta_{j l}$,

$\theta_{i j}=\frac{1}{c_{j}}$.

Since we assume that each terminal can have a wireless connection with only one base station, we use the following update function to keep one firing for each terminal,

$x_{i j}(t+1)=\left\{\begin{array}{cc} & \text { when } y_{i j}(t+1)=\max \left[y_{i 1}(t+1),\right. \\ & \left.\ldots, y_{i n}(t+1)\right], \\ 0 & \text { otherwise },\end{array}\right.$

$y_{i j}(t+1)=\sum_{k=1}^{m} \sum_{l=1}^{n} w_{i j k l} x_{k l}(t)-\theta_{i j}$

By updating the neurons by Eqs. (15) and (16) with the connections and thresholds in Eqs. (13) and (14), the state of the neural network converges to the minimum of Eq. (12), which corresponds to the optimal state of the base station selection.

Examples of simulation results are shown in Figs. 5 and 6. In this simulation, 1000 base stations are located in a square field whose size is varied. In order to check effectiveness of the proposed scheme, the average throughput and the fairness of the throughput are evaluated. For the fairness, Jain's fairness index [18] is used. For the comparison, general distributed base station selection schemes, random selection, nearest base station selection and capacity-based selection are also shown.

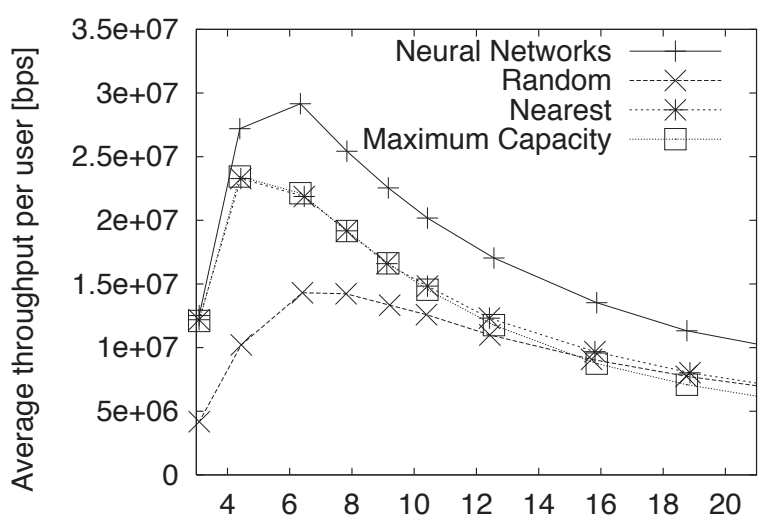

Average number of available base stations

Figure 5. Experimental results on the average throughput of the distributed load balance optimization implemented on a heterogeneous cognitive wireless network system.

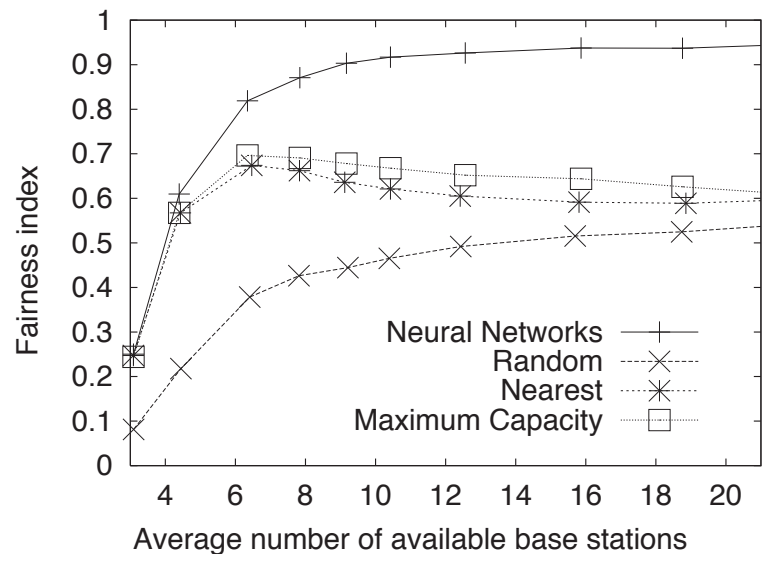

Figure 6. Experimental results on the fairness index of the distributed load balance optimization implemented on a heterogeneous cognitive wireless network system.

The graphs clarify that the proposed neural selection can improve the highest throughput and the highest fairness. Since distributed implementation of the neuronal update is possible, this neural approach can be applied to the case that a centralized server is not available or not prepared in the networks. By the corporative exchange of the state of neurons among the updating devices, each device can select the best resource without any centralized management.

The optimization problem, which we have dealt with in this paper, is very simple and its objective function could be transformed to the 2 nd order function of the neural network state $x_{i j}$, the same order as the original Hopfield-Tank neural networks. For more higher-order objective function, we can introduce the high-order neural networks [19], which autonomously decreases higher-order energy function by distributed neuronal updates. We have already applied such higher-order neural network in Ref. [20] to higher-order optimization problems in distributed cognitive radio networks. 


\section{CONCLUSION}

In this paper, we have defined optimization algorithms for heterogeneous cognitive wireless networks. As a typical optimization problem, we introduced load balancing to improve the service quality of the entire wireless networks. To optimize the problem, we propose two algorithms. For the network which can be managed by a centralized server, we have realized an rigorous optimization algorithm, by modeling the problem as a minimum cost flow problem. For the network which cannot be managed by a centralized server, we apply a distributed algorithm based on the Hopfield-Tank neural network.

We have dealt with a problem in heterogeneous wireless networks, traffic load balancing, which is a typical optimization problem to avoid congestion of the traffic. In order to improve the radio resource usage of the wireless networks, there are various other factors, which should be optimized. In this paper, we showed two examples how to optimize the network. The proposed optimization framework can be also applied to various more complicated optimization problems in wireless networks. Further, we have implemented the proposed algorithms on an experimental wireless network, Cognitive Wireless Cloud system [21,22]. Using the implemented system, we have shown that the distributed algorithm works correctly by our design of protocol based on IEEE1900.4 architecture [23].

Our important future work is to realize a general optimization algorithm, which can be applied to the cognitive radio system including the estimated function $f(\mathbf{x})$ as defined in Fig. 1. Although this paper focused on a predefined static optimization problem, relation between the action and the performance improvement $f(\mathbf{x})$ has to be learned based on the experiences in more general cognitive radio systems. As an example of such cognitive radio systems optimizing the decision based on estimated $f(\mathbf{x})$ as in Fig. 1, we have applied a full search for the throughput maximization of network aggregation in Ref. [24]. We are working toward development of more efficient optimization algorithms for the function estimated by learning algorithms in the cognitive radio system.

\section{REFERENCES}

[1] J. Mitola and G. Q. Maguire, "Cognitive Radio: Making Software Radios More Personal," IEEE Personal Communications, vol. 6, no. 4, pp. 13-18, Aug. 1999.

[2] S. Haykin, "Cognitive Radio: Making Software Radios More Personal," IEEE Journal on Selected Areas in Communications, vol. 23, no. 2, pp. 201-220, Feb. 2005.

[3] S. Haykin, "Cognitive Dynamic Systems: Radar, Control, and Radio," Proceedings of the IEEE, vol. 100, no. 7, pp. 2095-2103, Jul. 2012.

[4] L. Giupponi, R. Agusti, J. Perez-Romero, O. Sallent Roig, "A Novel Approach for Joint Radio Resource Management Based on Fuzzy Neural Methodology," IEEE Trans. on Vehicular Technology, vol. 57, no. 3, pp. 1789 - 1805, May 2008.

[5] K. Tsagkaris, G. Dimitrakopoulos, A. Saatsakis, P. Demestichas, "Distributed Radio Access Technology Selection for Adaptive Networks in High-Speed, B3G Infrastructures", International Journal of Communication Systems, vol. 20, no. 8, pp 969 - 992, Aug. 2007.

[6] X. Yue, C. M. Wong and S. G. Chan, "CACAO: Distributed Client-Assisted Channel Assignment Optimization for
Uncoordinated WLANs," IEEE Trans. Parallel and Distributed Systems, vol. 22, pp. 1433 - 1440, 2011.

[7] N. Baldo and M. Zorzi, "Cognitive Network Access using Fuzzy Decision Making," IEEE Trans. Wireless Communications, vol. 8, pp. 3523 - 3535, 2009.

[8] R. Xie, F. R. Yu and H. Ji, "Dynamic Resource Allocation for Heterogeneous Services in Cognitive Radio Networks With Imperfect Channel Sensing," IEEE Trans. Vehicular Technology, vol. 61, pp. 770 - 780, 2012.

[9] IEEE Std. 1900.4, IEEE Standard for Architectural Building Blocks Enabling Network-Device Distributed Decision Making for Optimized Radio Resource Usage in Heterogeneous Wireless Access Networks, 2009.

[10] J. J. Hopfield and D. H. Tank, "Neural Computation of Decisions in Optimization Problems," Biological Cybernetics, vol. 52, no. 3, 141 - 152, Jul. 1985.

[11] IEEE P802.11af, "Wireless LAN in the TV White Space," http://www.ieee802.org/11/Reports/tgaf update.htm

[12] IEEE Std 802.22.1-2010, “IEEE Standard for Information Technology-Telecommunications and information exchange between systems-Local and metropolitan area networksSpecific requirements Part 22.1: Standard to Enhance Harmful Interference Protection for Low-Power Licensed Devices Operating in TV Broadcast Bands," Nov. 2010.

[13] IEEE Std 802.16h-2010, "IEEE Standard for Local and metropolitan area networks Part 16: Air Interface for Broadband Wireless Access Systems Amendment 2: Improved Coexistence Mechanisms for License-Exempt Operation," July 2010.

[14] D. P. Bertsekas and P. Tseng, "Relaxation methods for minimum cost ordinary and generalized network flow problems," Operations Resaech, vol. 36, pp. 93-114, Jan. 1998.

[15] M. D. Grigoriadis, "An efficient implementation of the network simplex method," Mathematical Programming Studies, vol. 26, 83-111, 1986.

[16] J. L. Kennington and R. V. Helgason, "Algorithms for Network Programming," Wiley, New York, 1980.

[17] A. V. Goldberg, "An Efficient Implementation of a Scaling Minimum-Cost Flow Algorithm," Journal of Algorithms, vol. 22, no. 1, pp. 1-29, Jan. 1997.

[18] R. Jain, D. Chiu and W. Hawe, "A Quantitative Measure of Fairness and Discrimination for Resource Allocation in Shared Computer System," DEC Technical Report, 301, 1984.

[19] B. S. Cooper, "Higher order neural networks for combinatorial optimisation improving the scaling properties of the Hopfield network," Proc. IEEE International Conference on Neural Networks, pp. 1855 - 1860, Nov. 1995.

[20] M. Hasegawa et al., "Application of Higher Order Neural Network Dynamics to Distributed Radio Resource Usage Optimization of Cognitive Wireless Networks," Lecture Notes in Computer Science, vol. 5506, pp. 851 - 858, 2009.

[21] H. Harada et al., "Research and Development on Heterogeneous Type and Spectrum Sharing Type Cognitive Radio Systems," Proc. of International Conference on Cognitive Radio Oriented Wireless Networks and Communications, Jun. 2009.

[22] K. Ishizu et al., "Design and Implementation of Cognitive Wireless Network based on IEEE P1900.4," Proc. IEEE Sensor, Mesh and Ad Hoc Communications and Networks Workshop, Jun. 2008. 
[23] M. Hasegawa, H. Hirai, K. Nagano, H. Harada, K. Aihara, "Optimization for Centralized and Decentralized Cognitive Radio Networks," Proceedings of the IEEE, vol. 102, no. 4, pp. 574-584, 2014.

[24] Y. Kon, K. Hashiguchi, M. Ito, M. Hasegawa, K. Ishizu, H. Murakami, H. Harada, "Autonomous Throughput
Improvement Scheme Using Machine Learning Algorithms for Heterogeneous Wireless Networks Aggregation,", IEICE Trans, Communications, vol. E95-B, no. 4, pp. 1143-1151, Apr. 2012. 